\title{
Automotive cylinder heads: recent advances on Thermal- Mechanical Fatigue design and upcoming challenges
}

\author{
Fabien Szmytka ${ }^{1,4}$, Pierre Osmond ${ }^{2}$, Luc Rémy ${ }^{3}$, Pierre-Damien Masson ${ }^{2}$, Agathe Forré ${ }^{1}$, \\ ${ }^{1}$ Science and Future Technologies Department, Groupe PSA, Vélizy-Villacoublay, France \\ ${ }^{2}$ Chassis and Engine Material Department, Groupe PSA, La Garenne Colombes, France \\ ${ }^{3}$ Centre des Matériaux, Mines Paristech, UMR CNRS 7633, Evry, France \\ ${ }^{4}$ Institute for Mechanical Sciences and Industrial Applications (IMSIA-UME), ENSTA Paristech, Palaiseau, France
}

\begin{abstract}
Through the peculiar case of an automotive cylinder head, actual and forthcoming challenges for low-cycle and thermal-mechanical fatigue design in an industrial context are presented. A special focus is applied on variable loadings, constitutive models and their interaction with the environment, fatigue criteria and structure validations.
\end{abstract}

\section{Introduction}

Evolutions in environmental rules combined with the competitive race to produce cleaner and lighter engines have induced, since about twenty years, a constant increase in thermal fluxes applied to automotive engine parts such as cylinder heads. These loading conditions lead to a well-known serious risk of thermalmechanical fatigue (TMF) cracks initiation that must be controlled to design reliable structure.
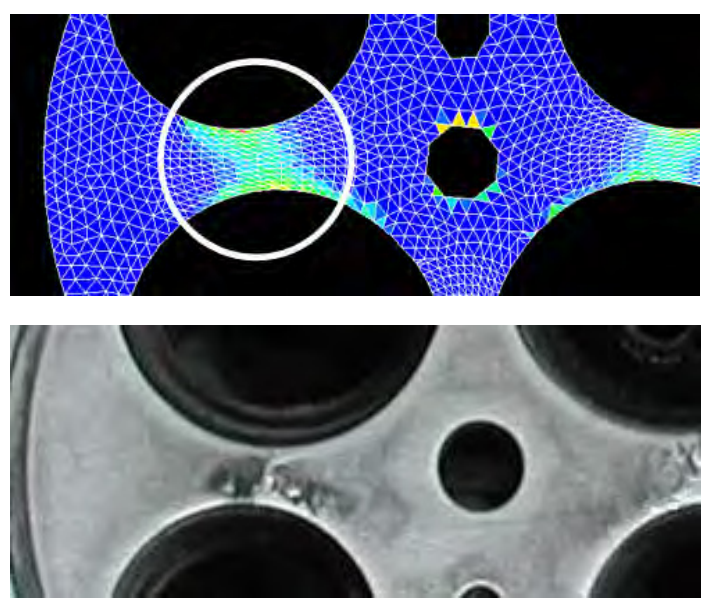

Fig. 1. Localisation of damage for cylinder under deterministic design loading: simulation (top) and experimental test (bottom)

Car manufacturers, and more particularly PSA Group have proposed many evolutions since Charkaluk and coworkers [1] developed the first protocol against TMF for automotive parts (with success in estimating critical zones and fatigue lifetime as seen on Figure 1), beneficiating of a better understanding of the structure behaviour, enhanced computation capacities, and better experimental investigation techniques. This article aims therefore at providing a summary of these improvements and of the upcoming challenges through the example of the cylinder head.

First, the randomness of the thermal loadings is presented as a crucial point for this part fatigue design. For a long time, deterministic design protocols have been used but their high level of conservatism tends to disqualify them today. Huge efforts have been recently made to successfully propose probabilistic design methods, relying on the study of loading and material TMF resistance randomness. Many statistical hypotheses are still questioned and will be briefly discussed. Moreover, aluminium alloys ageing which are of primary importance for the fatigue design, are deeply influenced by these loading sequences. Some reflexions about this phenomenon's impact on TMF resistance evaluation will also be proposed.

In a second time, constitutive models improvements by using inspired-by-physic equations relying on dislocations densities are proposed. Micro-crack growth laws and defects population are introduced in fatigue criterion and the impact on the fatigue design protocol is then evaluated. The reliability of these two elements is presented as crucial for proposing non-linear damage accumulation strategies, more appropriate for probabilistic design protocols. Simplified methods for numerical integration of such models leading to a more flexible use of complex equations are jointly presented.

Finally, a peculiar attention is dedicated to an experimental set-up based on a simplified structure (single cylinder head), half the way from specimens to real industrial structures. They enable to reproduce the complexity of real loadings on structure for which the strain and stress analyses are far easier. Example of 
comparison between models and experiment is detailed to underline the previously discussed improvements.

\section{Material}

Cylinder heads are currently made from aluminium alloys containing silicon, magnesium and often copper due to their high strength to weight ratio, high thermal conductivity, good casting properties as well as machinability. The A356 alloy is widely used and strengthened by precipitation (T7 heat treatment). Alloys containing copper are also widely used in the automotive industry like the A319 alloy [2]. Many cylinder heads are nowadays produced by die casting. Meanwhile, the lost foam casting process was recently introduced to further reduce the processing cost while opening geometry optimisation new opportunities. However, the slower cooling rates result in coarser microstructures and larger porosity as presented for an A356-T7 alloy on Figure 2.

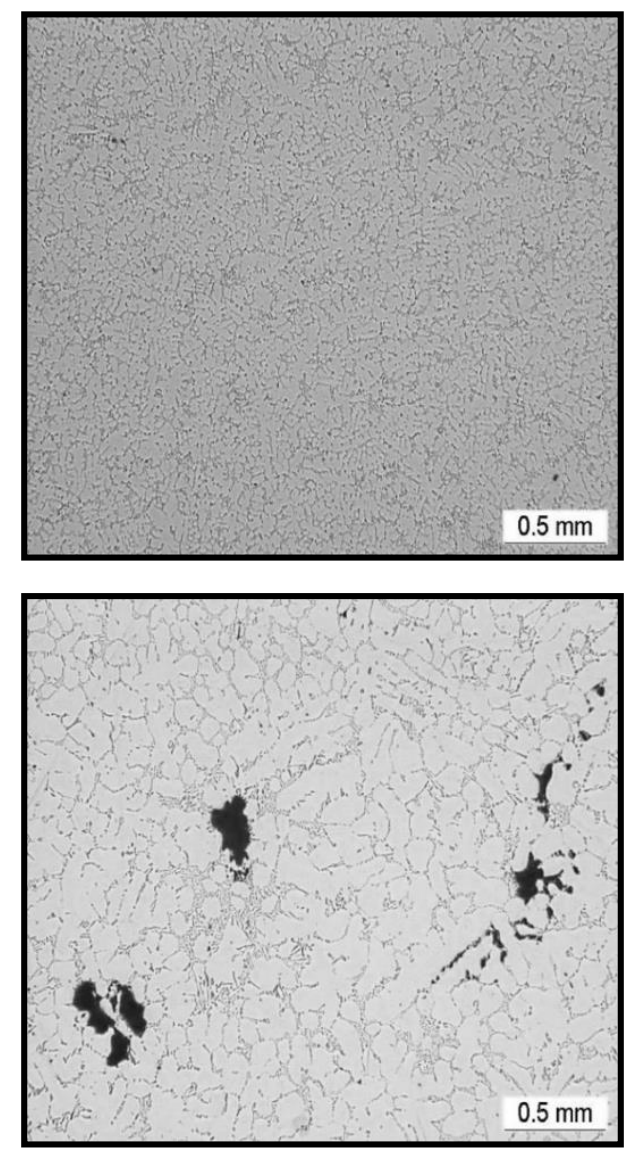

Fig. 2. A356-T7 alloy microstructure for die casting (on top) and lost foam casting (bottom)

The design protocol used today has been established for A356-T7 aluminium alloy that is widely used for PSA cylinder heads in diesel common rail injection engines [1]. Such components can experience operating temperatures that can exceed $250^{\circ} \mathrm{C}$ in some critical areas. Maximum temperatures thus can exceed the temperature for the precipitation treatment so that overageing occurs during service. That results in a loss of strength and of fatigue resistance. In long term overaged condition, this alloy exhibits a single residual micro-hardness, essentially independent of temperature, as shown on Figure 6- and presented in [3].

\section{Variable Loadings and Reliable design}

For a long time, deterministic design protocols have been used but their high level of conservatism tends to disqualify them today. Huge efforts have been recently made to successfully propose probabilistic design methods, taking account of the loading randomness, which is a key point for fatigue. If one wishes to estimate the variability in the use of a structure by a given population and the possible dispersion at which may be subject the resistance of this same structure, it is fundamental to experimentally measure this variability and therefore to collect as much information as possible by establishing experimental databases. These latter will then contribute to the improvement of constitutive models and fatigue criterion as mentioned in the following sections.

Measuring the "stress" applied to an automotive structure subjected to thermomechanical fatigue consists in building a distribution of severity of use, taking into account both the usage patterns (road type, vehicle embedded mass, weather conditions, etc.) but also the severity with which a driver will use his vehicle. It is therefore necessary to analyse and measure these two aspects of the "stress" environment. To simplify the problem, one can limit the usage patterns at the travelled types of roads. In order to obtain reliable information on their habits, surveys are conducted on a large population of vehicle drivers. One then classes the vehicle usage according to specific usage criteria such as "Classic Road", "Highway", "City" or "Mountain". It is asked to the vehicle owner to estimate the percentage of time spent or total mileage corresponding to each type of road during a year. This enables to create a customer database in terms of vehicles usage.

To be able to know the vehicles' usage conditions is far from sufficient to estimate a severity of use. Indeed, it is also essential to know the conditions of loading of this structure we want to design on all the specific types of roads, while taking into account the driver behaviour variability. In order to estimate both the drivers' behaviour and structure loads for a specific type of road, the structure one want to design has to be equipped with sensors. This vehicle is then driven on multiple courses taken as references for every possible "real-life" situations by a panel of professional drivers. This produces a matrix of elementary life situation of loading for the vehicle and by the way the studied structure, mixing both road and driver types and helps to understand the loading environment on the structure to be designed and then to adapt the design protocol.

The available data to build the probability density for customers' loads are macroscopic records for overall level of loading (engine speed and torque). However, designing cylinder heads for thermomechanical fatigue is 
linked to the description of a local phenomenon on the fire deck intervalve bridge and most usually to a finite element analysis in order to quantify the damage per cycle [1]. To take into account the usage variability, one needs to estimate local mechanical answer from global and monitored loadings. Signal processing methods are then developed to exhibit basic loading cycles from global loading signals [4]. In the same time, a global/local transition law is built to estimate the local damage resulting from one global basic loading cycle.

The first step is to choose an observable variable to represent the global loading condition. The relevant boundary condition for a FEM analysis is the thermal flux density applied on the cylinder head fire deck, which can easily be extracted from the engine speed and torque monitoring, coupled with the engine combustion parameters. An engine load is then defined as a random non-zero temporal evolution of thermal flux density applied on the fire deck. The development of a signal processing method, based on the principle of "thermomechanical loading", enables to characterize a material for TMF studies by taking into account the nonlinear parts of its behaviour. This step is in most cases realised with elasto-viscoplastic models where hold times, strain or temperature amplitudes and rates are the most important parameters.
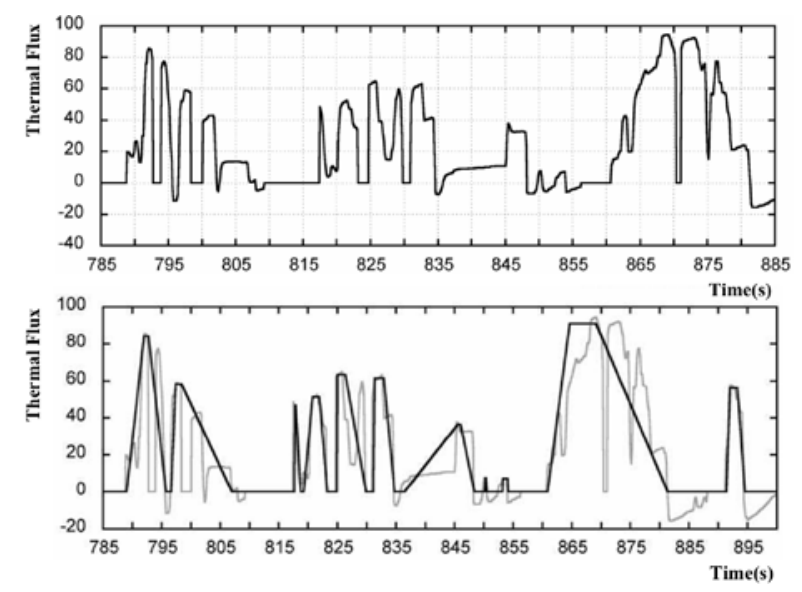

Fig. 3 Real loading sequence for a cylinder head (on top) and filtered load sequence (bottom).

Global temporal signals are thus filtered following the principle described on Figure 3. One aims at detecting cycles which main characteristics are flux variation rates, hold times for high and low flux values and maximal and minimal flux values. The signal processing principles also enable to take into account the thermal inertia of the cylinder head and thus in particular to group basic identified cycles closely spaced together in time and for which the quick flux variations lead only to very limited fire deck temperature variations. It is thus possible to obtain, from a complex and noisy signal, a succession of basic loading cycles as seen once again in Figure 3.

Here, it becomes obvious that deterministic design protocols relying on the succession of standardized severe loading cycles are far from the experimental reality. Mastering the loading conditions is the first step to optimize the fatigue design. Constitutive models and fatigue criteria are the two other ones.

\section{Constitutive model and ageing conditions}

A common design procedure used to handle the problem of ageing during service is to assume two different constitutive cyclic (and monotonic) stress-strain behaviours. A conventional viscoplastic Chaboche model [5] is identified for initial T7 condition and used for low temperature areas. A second Chaboche model is identified for the long-term overaged condition for areas experiencing high temperatures (Figure 4). However, when casting conditions are changed, when the loading conditions become very variable or when alloy composition is modified, one needs to assess the validity of the present design procedure, or to change it if necessary.
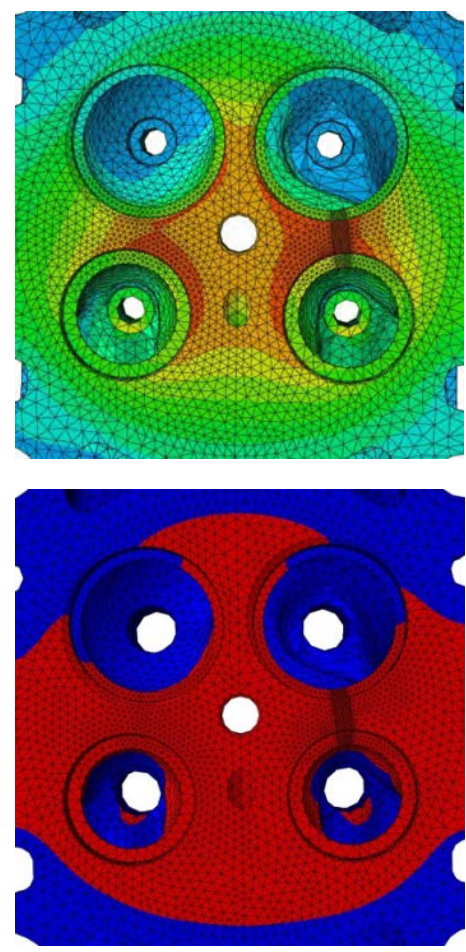

Fig. 4 Example of thermal map for a fire deck and localisation of an "overaged" zone

Figure 5 shows the different scales of microstructure. During the last decade, the influence of variations in compositions and overageing treatments conditions has been investigated. Conventional micro-hardness measurements were combined with transmission electron microscopy observations to quantify the variations in precipitate distribution (nature of precipitates, size and volume fraction). Some specific overageing conditions were selected and cyclic stress-strain behaviour was identified at different temperatures. 
To establish the link between heat treatments and microstructure variation, a microstructure sensitive model was used instead of a Chaboche one. Basically, the model uses dislocation density as internal variable, considering dislocation multiplication and dynamic recovery as proposed by Kocks and Estrin ([6], [7]). Static recovery can be easily introduced and this type of model was shown to be very effective for stainless steels used in exhaust manifolds [8]. Dislocations by-passing precipitates by Orowan mechanism leave loops around precipitates, which is handled by specific contributions in isotropic and kinematic hardenings [9].

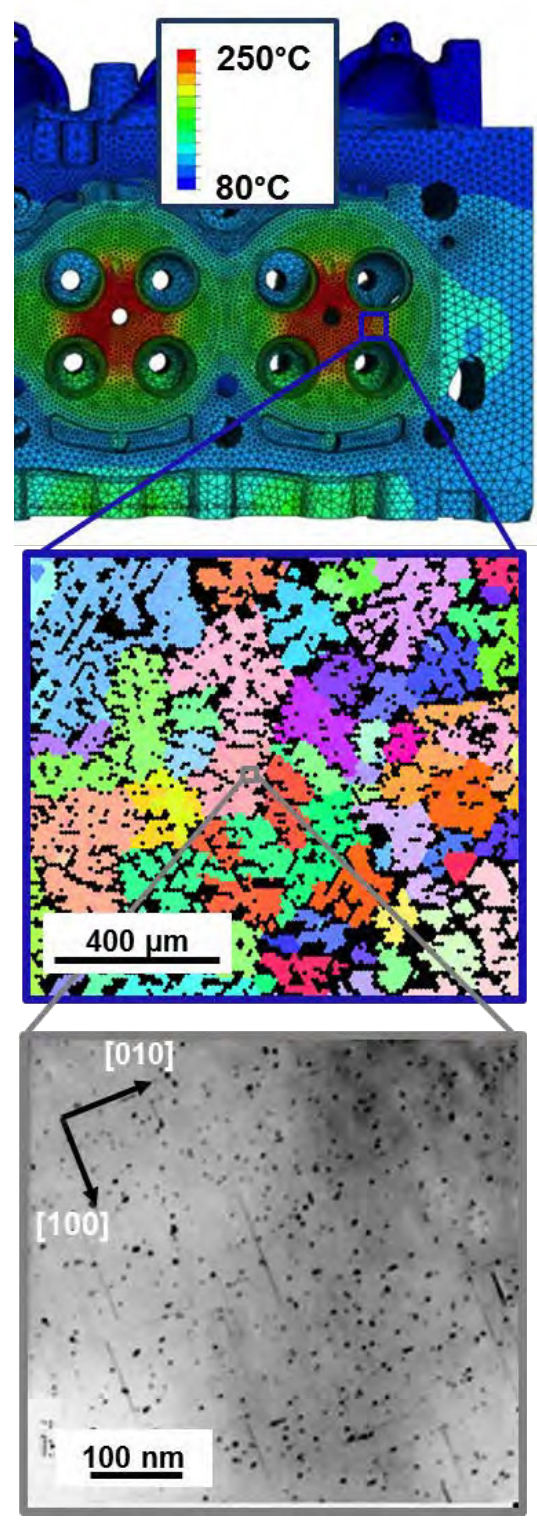

Fig 5: Temperature map on cylinder head (on top), typical grain microstructure of A356-T7 aluminium alloy (middle) with typical strengthening precipitation in $\mathrm{T} 7$ condition inside a dendrite (bottom).

This procedure combines physical constants coming from literature and dislocations models, microstructure parameters from TEM observations and optimised parameters from experimental cyclic stress-strain loops. Once these parameters are identified from a series of overageing treatments, they can be used on a range of alloy composition and aging conditions. The model has been validated using out-of-phase thermal mechanical tests [9].
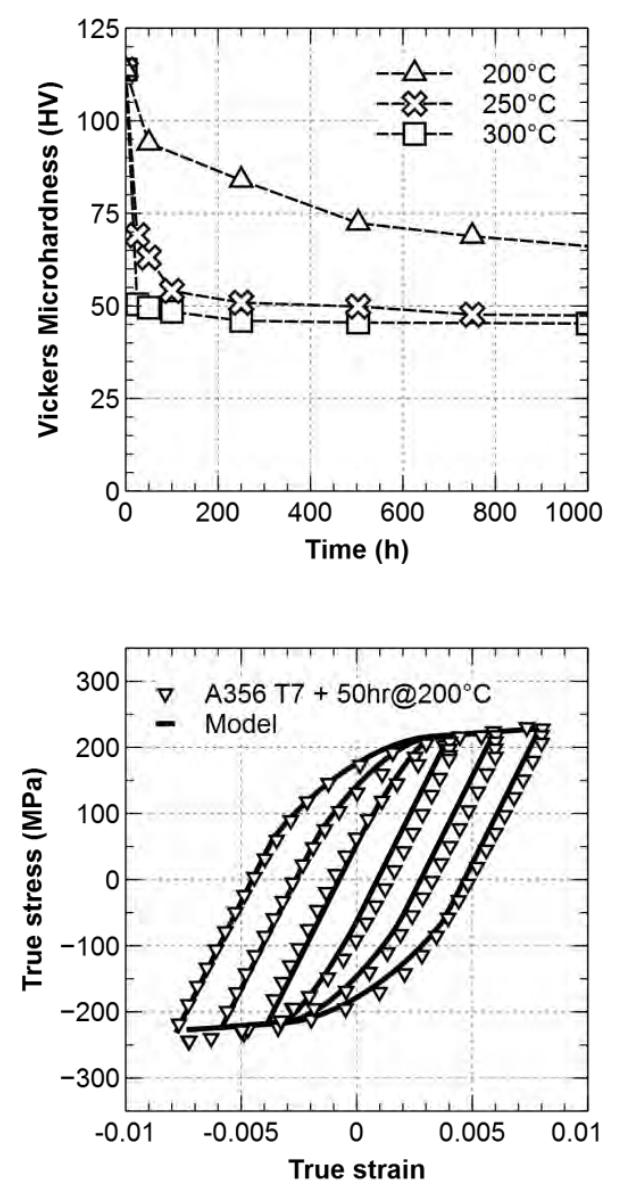

Fig. 6: Change in Vickers microhardness during isothermal ageing (top); Model versus experimental cyclic stress-strain loops for an intermediate overageing treatments (bottom).

Next step is to apply this model to the microstructure gradient that occurs after long-term service operation on a cylinder head. This was tested using an experimental protocol presented above. Depending upon alloy composition, the current design procedure can give rather conservative prediction of fatigue life compared to the improved one [10] but this latter implies longer computation times. Future development must therefore focus on numerical strategies to offer predictive thermal evolution with limited CPU times.

\section{Fatigue criteria}

Fatigue data are most of the time obtained on overaged materials to ensure stable mechanical behaviour even if progressive ageing coupled with variable amplitude loading may lead, in the future, in an evolution of standard fatigue tests. A full 3D characterization of the microstructure through X-ray tomography [11] can then be performed in order to measure the size and shape distribution of pores in a Representative Elementary Volume. 

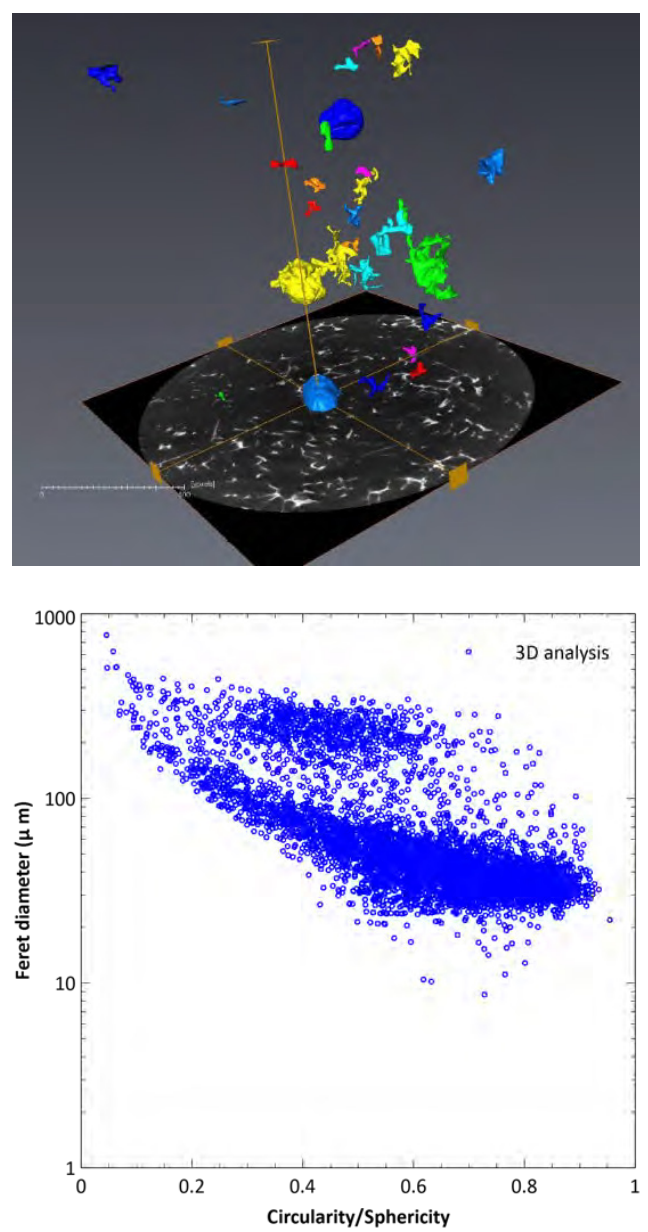

Fig. 7: Example of pore detection by X-ray tomography for a A356 lost foam cast alloy and pore population ranked by size and sphericity.

A quantitative description of the pore population is obtained through the measurement of their Feret diameter and sphericity, which could reveal the presence of gassing porosities for lost foam casting processes which are not observed in standard die casting processes. The largest and sharpest pores have to be accurately observed as they play an important part in the fatigue crack initiation. The pores size distributions are then introduced in the computational approach used to design engine parts against TMF. Under a basic assumption of growth equation of pores from an initial size $a_{0}$ to a final size $a_{f}$ at $N_{f}$ characterizing a macroscopic crack initiation, a fatigue criterion is developed under a probabilistic form as proposed by [12]. Different damage indicator, as proposed by $[13,14]$, can enable to take into account micro-cracks propagation and leads to the following improved form of the fatigue criterion:

$$
N_{f}=\int_{a_{o}}^{a_{f}} \frac{1}{\left[\left(\frac{w^{d}}{k_{1}}\right)^{n_{1}} a^{\left.n_{1}+\left(\frac{w^{e}}{k_{2}}\right)^{n_{2}} a^{n_{2}}\right]}\right.} d a
$$

where $w^{d}$ and $w^{e}$ denotes respectively inelastic and elastic dissipated energy densities per stabilized cycle, and $k_{1}, n_{1}, k_{2}$ and $n_{2}$ are material constants. $a$ consists in the observed statistical distribution of pore size while material parameters are identified from the macroscopic
Low-Cycle Fatigue and TMF experiments. $N_{f}$ and $a$ are thus random variables, which is very important when dealing with variable loading amplitudes.
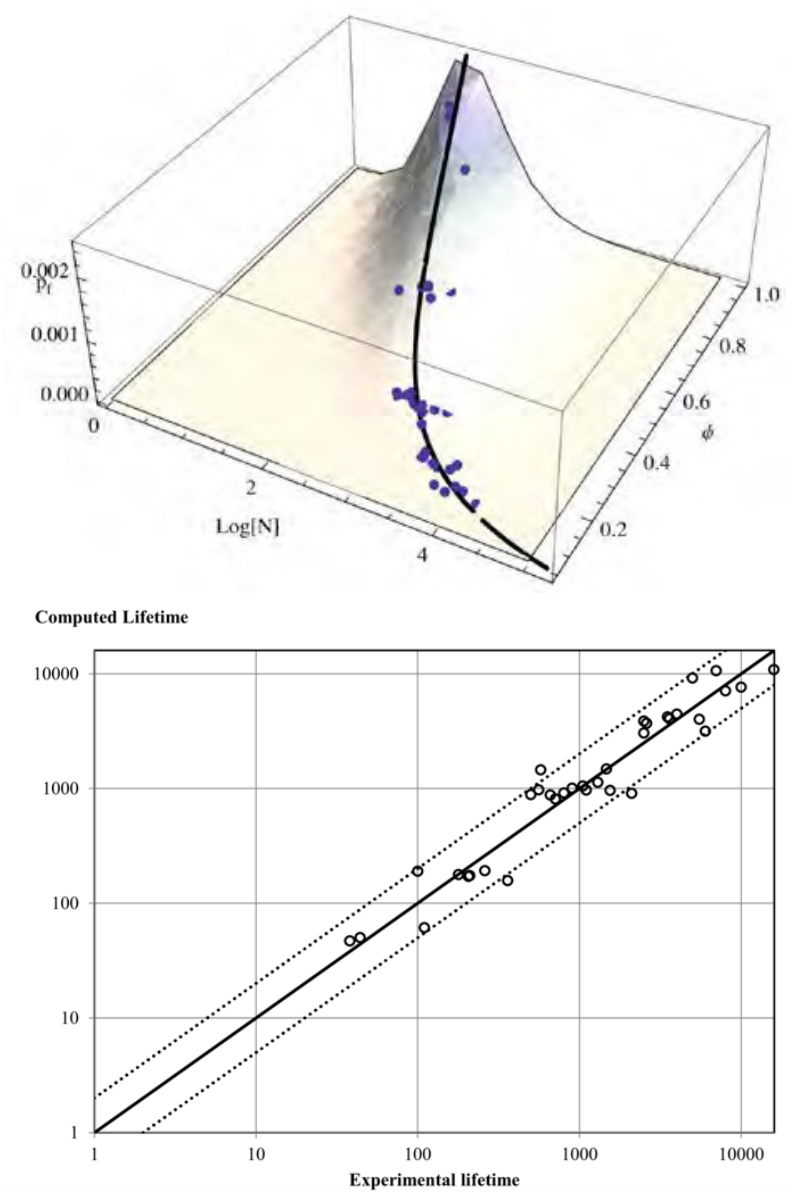

Fig. 8: Number of cycles before failure distribution as computed by [11] and Computed lifetime in terms of maximal probability of failure versus experimental lifetime for à lost foam cast A356 alloy.

When crack propagation is investigated on laboratory specimens, a direct access to the parameters is possible. In other cases, the simple numerical identification of the parameters of such a model is quite complex because no analytical description is possible. While experimental data are missing, treatment is then carried out for class of pore size. For a given pore size and for a given set of parameters $\left(k_{1} ; n_{1} ; k_{2} ; n_{2}\right)$, the estimated lifetime is easily calculated. A histogram and a complete distribution of the number of cycles to failure are then obtained. For a given experimental couple $\left(w^{d} ; w^{e}\right)$, the number of cycles to failure with the highest probability is then finally compared to the experimental lifetime. A least-squares optimization is then performed to obtain the most relevant parameter set. Lifetime prediction results can then be compared with laboratory specimens. One can therefore easily perform non-linear damage accumulation for variable amplitude loading cycles. Following this simple principle, good matching is obtained between estimated and experimental lifetimes as seen on Figure 7. Developments on crack growth law with generalized plasticity and on interactions between microstructural defects and fatigue lifetime are therefore 
of primary importance to produce robust criteria in the future.

\section{Industrial specimens experimental protocols}

Thermomechanical fatigue tests on laboratory specimen $[15,16]$, for the sake of analysis simplicity, introduce simplifications with respect to the effective loadings recorded on real structures. Thus, the thermal gradients, which are mainly responsible for damage by thermomechanical fatigue, are suppressed in the experimental protocol for the sake of proposing a direct analysis of the stress-strain curve. When these gradients become very severe, it is most of the time impossible to disregard them in an objective fatigue analysis. In these cases, it then becomes necessary to develop specimens closer to the structure to be designed and therefore often more complex in terms of geometry and loading [16, $17]$.

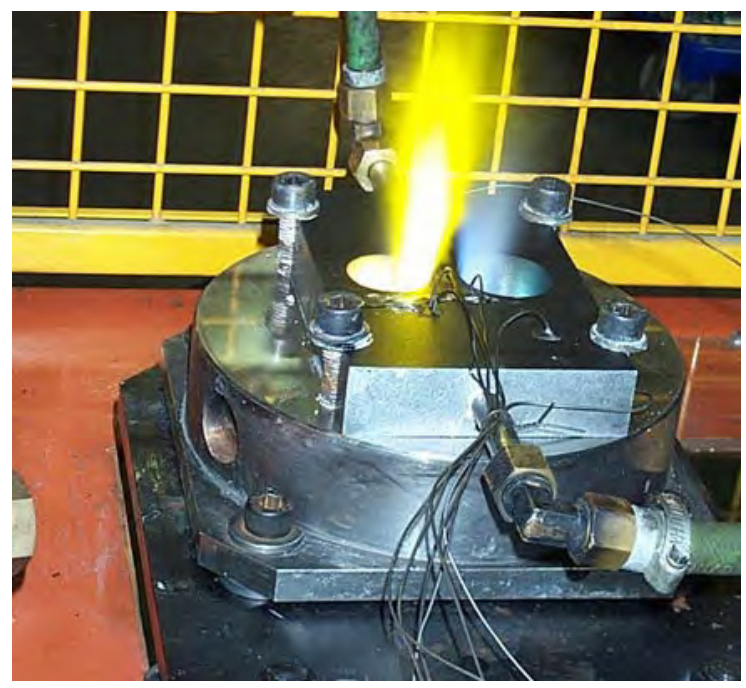

Fig. 9: Single cylinder thermal fatigue rig with an acetylene torch heating system

For cylinder heads, Constantinescu and co-authors [1] have proposed using massive specimens for which the microstructure or the surface roughness is not always perfectly representative of the structure to be designed. Thus, the development of a "single-cylinder" specimen as seen on Figure 8 enables loadings as close as possible to the structure ones while respecting both the microstructure and the surface roughness in the critical zone. Development of such rigs is of primary importance in the fatigue design protocols as they enable a precise analysis of the fatigue strength and the validation of constitutive models and fatigue criteria.

\section{Conclusions}

Taking into account in-use evolutions of thermal and mechanical loadings and thus their variability constantly modifies the way of designing structures against thermomechanical fatigue. The use of simplifying assumptions to take into account aluminium alloys mechanical behaviour and overageing is now clearly inconsistent with the need to produce reliable and robust design protocols. In fact, ensuring reliable and predictive fatigue design has to rely on a careful analysis of the boundary loading conditions, a reliable constitutive model taking into account the physical evolution linked to these loadings and advanced criteria including real damage mechanisms. Finally, development of simplified testing rigs closer to the inuse loadings and to standard production parts process conditions now seems essential to validate models and improve designs.

\section{References}

1. A. Constantinescu, E. Charkaluk, G. Lederer, L. Verger, International Journal of Fatigue, 26 (2004) 805-814

2. H. Sehitoglu, X. Qing, T. Smith, H.J. Maier, J. A. Allison, Metallurgical and Materials Transactions A, 31A (2000) 139-151

3. S. Tabibian, E. Charkaluk, A. Constantinescu, G. Guillemot and F. Szmytka, MSEA, 646 (2015) 190200

4. F. Szmytka, F., A. Oudin, International Journal of Fatigue, 53 (2013) 82-91

5. J.L. Chaboche, International Journal of Plasticity, 24 (2008) 1642-1696

6. U.F.Kocks, A.S.Argon, M.F.Ashby, Progress in Materials Science, 19 (1975), 110-170

7. Y.Estrin, Journal of Materials Processing Technology, 80 (1998) 33-39

8. L. Rémy, F. Szmytka, L. Bucher, International Journal of Fatigue, 53 (2013) 2-14

9. P. Osmond, Ph. D. Thesis (in French) Mines ParisTech (2010)

10. F. X. Hoche, L. Remy, L. Naze, P. Osmond, A. Koster, This conference

11. N. Limodin, A. El Bartali, L. Wang, J. Lachambre, JY Buffiere, E. Charkaluk, Nuc. Instru. and Meth. in Phy. Res. B, 324 (2014) 57-62

12. E. Charkaluk, A. Constantinescu, F. Szmytka, S. Tabibian, International Journal of Fatigue, 63 (2014) 127-136

13. L. Rémy, N. Haddar, A. Alam, A. Koster, N. Marchal, MSEA, 468-470 (2007) 40-50

14. V. Maurel, L. Rémy, N. Haddar, F. Damen, International Journal of Fatigue, 31 (2009) 952-961

15. J.L. Malpertu, L. Rémy, Metall. Trans. A, 21A (1990) 389-399

16. L.Rémy in Comprehensive structural integrity, $\mathbf{5}$ (2003) 113-200

17. F. Rezai-Aria, B. Dambrine, L. Rémy, Fat. Fract. Eng. Mat. Structures, 11 (1988) 291-302 